\title{
Physical and Mathematical Modeling of Steel Flow and Heat Trans- fer in Tundishes under Non-isothermal and Non-adiabatic Conditions
}

\author{
J. de J. BARRETO S., M. A. BARRÓN MEZA'1) and R. D. MORALES ${ }^{1)}$ \\ Graduate Center, Instituto Tecnológico de Morelia, Morelia Michoacán, México. \\ 1) Instituto Politécnico Nacional-ESIQIE, Department of Metallurgy, Apdo. Postal 75-874, México D. F. CP 07300.
}

(Received on October 6, 1995; accepted in final form on January 18, 1996)

\begin{abstract}
Steel flow and heat transfer in tundishes under non-isothermal and non-adiabatic conditions are physically and mathematically modeled. For this purpose thermal step inputs in both types of models are applied and temperature measurements as well as computations of flow and heat transfer at unsteady state are carried out.

Experimental measurements of output responses to thermal step inputs fed in the tundish model and the transient solution of the three dimensional Navier-Stoke's equations keep a very good agreement.

Isothermal lines of water model and those belonging to the prototype under non-adabatic conditions are not equivalent for the same thermal step input and this promotes different velocity fields of water in the model and liquid steel in the prototype.

As velocity fields have a direct influence on inclusions removal from steel to the covering tundish slag it is recommended to have caution on the employment of non-isothermal water models to interpret the actual behavior of steel flow and inclusions dynamics in industrial tundishes.
\end{abstract}

KEY WORDS: tundish; steel flow; heat transfer; non-isothermal; non-adiabatic; water models; mathematical models; inclusions; $k-\varepsilon$ model.

\section{Introduction}

The temperature of liquid steel during the continuous casting is one of the basic indicators of operation and quality control. In the past decade and during the present one, the requirements for high quality steel have increased dramatically, with more emphasis on superheat control and an ever need for better automation.

It has been long recognized that superheat plays a key role in determining the structure and properties of continuously casted products, therefore to achieve quality and easy of operation, the casting temperature must be controlled as closely as possible.

Unfortunately, ladle changing, heat losses from the ladle and the tundish and start up operations promote the non-isothermal behavior of steel in the tundish affecting its flow owing to differences on its density and the consequent generation of floating forces. This brings about changes on the average residence time of steel in the tundish affecting, also, the opportunity that the liquid steel has to take intimate contact with a refining and covering slag which may be able to trap non-metallic inclusions with a further steel refining.

To understand the steel flow in tundishes, many researchers have employed water models under isothermal conditions and the technical literature is plenty of reports with this approach. ${ }^{1-5)}$ On the other hand, mathematical models, with the same purposes, have been reported explaining experimental observations of water models under isothermal conditions. ${ }^{6-8)}$

In spite of all these efforts, few works have attempted to model physically and mathematically the steel flow under non-isothermal conditions. In the case of physical models there are some proposed similarity thermal criteria from which the experimental results may be used to interpret the actual steel flow in a given industrial tundish. ${ }^{9,10)}$

Among the few reports on the matter there are those of Sahai, ${ }^{11-13)}$ where, a three dimensional mathematical model for steel flow under non-isothermal behavior is applied are well known.

In the present work physical and mathematical modeling of liquid steel in a continuous casting tundish under non-isothermal conditions are carried out. The main objectives are to establish the basis to interpret the actual liquid steel flow in tundishes through experimental data of water models with temperature changes at the inlet stream and to find out the usefulness of matching physical with mathematical models to understand the effects of step changes of input stream temperature.

\section{Experimental}

\subsection{Physical Model}

Kinematic similarity between prototype and model is ensured if geometric and dynamic similarities are observed. The principal forces to be considered in obtaining dynamic similarity in the tundish are inertial, gravitational, viscous and surface tension forces. The principal dimensionless groups which involve these forces are given by the numbers of Froude, Reynolds and Weber which involve all forces already mentioned. Absolute 
dynamic similarity requires that each of the dimensionless groups listed above have the same value in both, the model and the prototype. Due to the difference of the physical properties of water and molten steel, it is impossible to satisfy simultaneously all of requirements for similarity which apply to fluid flow in the same model of a given particular scale. Reynolds-Froude similarity requires a full scale model. The Weber-Froude similarity requires a model of 0.6 scale.

It has been demonstrated by Heaslip ${ }^{14)}$ that the Froude number can be satisfied at any scale in a tundish water model as long as all metering oriffices and fluid hydraulic heads in the system are varied in accordance with a single scaling parameter. In this work the Froude number was satisfied by employing a one sixth water model of a typical slab caster tundish, including the ladle collector and the mould submerged entry nozzle. The experiments are described in the following lines:

(1) The tundish model. A one strand model was employed in this work just for an easy operation of the conductivity cell, which is described below, to quantify the mixing characteristics of a tracer fed into the model. Important parameters for the model and the prototype are given in Table 1. A schematic representation of the experimental set up is shown in Fig. 1. A $6 \mathrm{~mm}$ thick perspex sheet was used to construct the water analogue model. The ladle collector nozzle was machined from perspex blocks, with $13 \mathrm{~mm}$ internal diameter, it was attached to a two way change valve. It was also equipped with a syringe injector to add the tracer to the incoming water stream. For the tracer detection a conductivity technique was used. For this purpose the tundish exit nozzle was made from perspex pipe with a $13 \mathrm{~mm}$ internal diameter. They were attached to the bottom of the tundish by a screw thread so they could be interchangeable.

Table 1. Parameters for model and prototype.

\begin{tabular}{lcc}
\hline \multicolumn{1}{c}{ Parameter } & Model & Prototype \\
\hline Tundish width & $0.13 \mathrm{~m}$ & $0.79 \mathrm{~m}$ \\
Tundish length & $1.18 \mathrm{~m}$ & $7.10 \mathrm{~m}$ \\
Tundish depth & $0.13 \mathrm{~m}$ & $0.79 \mathrm{~m}$ \\
Wall inclination & $9 \mathrm{deg}$ & $9 \mathrm{deg}$ \\
Vol. flow rate & $12 / \mathrm{min}^{-1}$ & $8200 \mathrm{~kg} \mathrm{~min}^{-1}$ \\
Ladle nozzle diameter & $13 \mathrm{~mm}$ & $80 \mathrm{~mm}$ \\
Mold SEN diameter & $13 \mathrm{~mm}$ & $80 \mathrm{~mm}$ \\
\hline
\end{tabular}

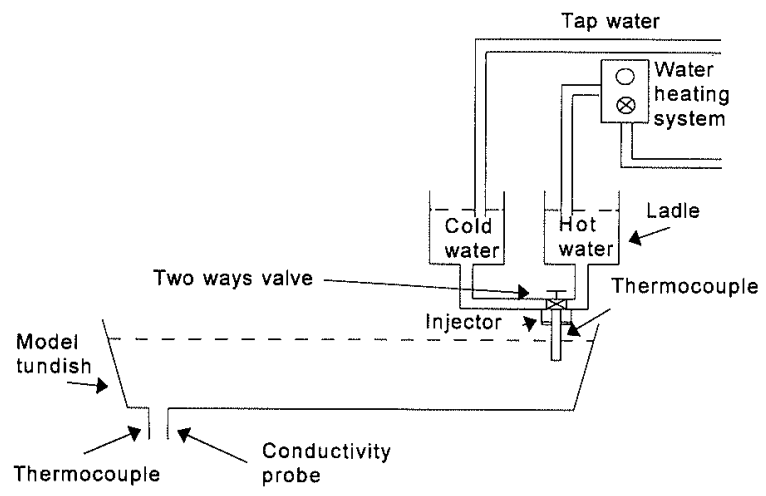

Fig. 1. Experimental set up.
(2) The ladle. The ladles were simple tanks of 20 litres capacity, supported above the tundish water level. One contained hot water and the other cold. Overflow pipes in the tanks allowed control of the water level. The ladles were connected by a two-ways interchangeable valve, so that water at changing temperatures could be supplied to the tundish model.

(3) Water heating system. One of the ladles contained hot water for that it was equipped with a water heating system. Because the hot water had to be supplied continuously, a $8 \mathrm{~kW}$ continuous electric heater was connected to the main water supply. This water was mixed with tap water before entering the tank by a "Y" junction, to make up the flow rate required.

The second ladle contained only tap water, which was supplied directly. The two ladles were connected by a two way diversion valve beneath them, and simply by changing its direction, hot or cold water was poured to the tundish through the sub-ladle entry nozzle. The valve was manually operated sufficiently fast to introduce a step input of temperature signal.

(4) The tracer and its preparation. Several materials have been used as tracers. Some of them are too dense and sink to the bottom of the tundish, flowing close to the lower surface towards the exit nozzle. Hydrochloric acid $(\mathrm{HCl})$ did not show that behavior and mixed well with the water flowing inside the tundish. Because, it is also conductive, it was chosen as the tracer for the conductivity measurements. The $\mathrm{HCl}$ used as a tracer was diluted to $90 \%$ with water. The solution was analyzed and the concentration found to be $9.3 \mathrm{~mol} \mathrm{dm}^{-3} ; 20 \mathrm{~cm}^{3}$ of this solution were injected by a syringe, as a pulse, at the injection point.

(5) Conductivity measurements. Platinum electrodes were attached on the inside walls, facing each other, close to the exit of the outlet nozzle, creating a conductivity cell. The electrodes were approximately half centimeter square and coated in platinum black. The cell was connected to a laboratory conductivity meter, and the output was recorded on an oscilloscope.

(6) Temperature measurements. Two Copper-Copper/Nickel, type " $T$ ", thermocouples were placed at the entry and outlet stream nozzles to measure the changes in temperature resulting both from changes in the temperature of the inlet stream and from the use of the heat system.

The flowing water in the tundish was left to get a steady state temperature before imposing a step input, of higher or lower water temperature, on the stream entry nozzle. The temperature of this sudden change was measured as it passed through the tundish nozzle by the thermocouples, and the output was amplified and recorded on an oscilloscope.

\subsection{Mathematical Model}

\subsubsection{Governing Equations}

The turbulent equations of change in the $k-\varepsilon$ model expressed in their three-dimensional version are employed in this work. These equations involve the following turbulent concepts; continuity, momentum, kinetic energy, dissipation rate of turbulence energy and 
Table 2. Physical properties of water and steel.

\begin{tabular}{lcc}
\hline \multicolumn{1}{c}{ Property } & $\begin{array}{c}\text { Water } \\
\text { (at } 293.15 \mathrm{~K})\end{array}$ & Steel \\
\hline Absolute viscosity & 0.001 & 0.005 (at $1873 \mathrm{~K}$ ) \\
Density & 1000 & $7010+\left(T-T_{\text {m.p. }}\right.$ ) $(-0.883)$ \\
Surface tension & 0.073 & 1.7 \\
Specific heat capacity & 4182 & 750 (at $1873.15 \mathrm{~K})$ \\
Thermal conductivity & 0.597 & 41 (at $1873.15 \mathrm{~K})$ \\
\hline
\end{tabular}

thermal energy. ${ }^{15)}$

At the walls of the tundish, including dams and weirs and the side wall of the entry and outlet nozzles the "logarithmic law wall"16) was used to correlate the velocity parallel to a given solid surface at the first nodal point adjacent to this surface and the shear stress applied at the wall. ${ }^{17}$

The values of the five constants in the $k-\varepsilon$ model are those recommended by Launder and Spalding. ${ }^{17)}$ The physical properties of water and liquid steel employed in this model are reported in Table 2.

\subsubsection{Initial and Boundary Conditions}

The boundary conditions for momentum transfer were those of non-slipping at the solid surfaces, zero normal velocity gradients at the symmetry planes and the free surface of the liquid. In a similar way, in the normal direction of the planes of symmetry both $k$ and $\varepsilon$ are given the condition of zero gradient, the values of these two variables at the entry point were assumed to obey already established empirical relations. ${ }^{17)}$

The initial conditions were settled by running the model for a given problem under some given specifications under adiabatic or non-adiabatic conditions for a step input of temperature until a steady state was reached. The velocity vectors in this state in all the three dimensional mesh were used as initial values to simulate the thermal effects of a step input.

In the case of calculation of temperature fields, two kinds of calculations were carried out in the prototype; adiabatic (no flow of heat through the walls of the tundish and free surface of the liquid) and non-adiabatic conditions. In the case of the water model, all calculations were adiabatic, since the energy lost from the tundish was assumed to be negligible compared with the sensible heat of water. Here, also, an initial uniform temprature at steady state in the three dimensional mesh was used to start a run with a step input.

In the case of non-adiabatic conditions heat fluxes through the tundish wall and the free surface of liquid were used as boundary conditions. Table 3 shows the selected values of these fluxes; the fluxes of heat at the planes of symmetry were assumed also to be zero.

The momentum and energy equations as well as all initial and boundary conditions were rewritten in a finite differences scheme, the three dimensional mesh employed in these simulations is shown in Fig. 2, and solved by a numerical technique called PISO ${ }^{18,19)}$ (Pressure Implicit with Splitting Operations). This is a time-marching procedure in which, for each time step, a predictor is followed by one or more corrector steps
Table 3. Parameters for mathematical simulation of nonadiabatic conditions.

\begin{tabular}{lc}
\multicolumn{1}{c}{ Parameter } & Value \\
\hline Volume of water in the model & $0.0137 \mathrm{~m}^{3}$ \\
Water flow rate in the model & $2.066 \times 10^{-4} \mathrm{~m}^{3} / \mathrm{s}$ \\
Mean time of water in the model & $66.29 \mathrm{~s}$ \\
Volume of steel in the tundish & $2.97 \mathrm{~m}^{3}$ \\
Steel flow rate in the tundish & $1.95 \times 10^{-2} \mathrm{~m}^{3} / \mathrm{s}$ \\
Mean time of steel in the tundish & $152.30 \mathrm{~s}$
\end{tabular}

Heat fluxes ${ }^{11}\left(\mathrm{~W} / \mathrm{m}^{2}\right)$ : Free surface, 15000 ; bottom, 1400 , Vertical walls: 3200 , transversal walls, 3800 .

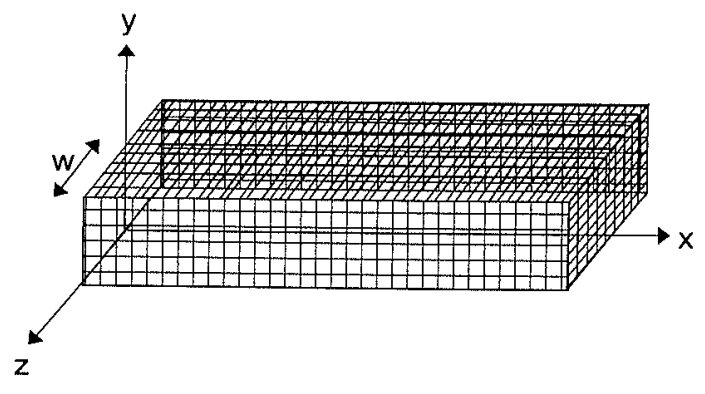

Fig. 2. Computational mesh used in the model (not at scale).

Table 4. Specific conditions of simulation runs by the mathematical model.

\begin{tabular}{lccc}
\hline \multicolumn{1}{c}{ Parameter } & $\begin{array}{c}\text { Water } \\
\text { model }\end{array}$ & Prototype & $\Delta T(\mathrm{~K})$ \\
\hline Number of strands & 1 & 1 & \\
Distance of weir from inlet & 0.770 & 4.623 & \\
Depth of weir in the fluid & 0.065 & 0.390 & \\
Distance of dam from inlet & 0.308 & 1.847 & \\
Height of dam & 0.065 & 0.390 & \\
Heat flux conditions & Adiabatic & $\begin{array}{c}\text { Adiabatic and } \\
\text { non-adiabatic }\end{array}$ & \\
Initial temperature & 295.15 & $\begin{array}{c}1841 \text { and } \\
1840^{*}\end{array}$ & 9 \\
& & 1831 & 9 \\
\hline
\end{tabular}

* This temperature corresponds to the steady state under nonadiabatic condiions.

using a non-iterative splitting of operations of discretized momentum and pressure equations. In this way, the fields obtained at the end of each step are close approximations of exact solutions of the turbulent change equations.

In order to obtain suitable graphs of the results of this mathematical model they were arranged in an appropriate format and fed to a commercial plotting program known as Tecplot. The specific conditions for the corresponding calculations carried out in this work are summarized in Table 4.

\section{Results and Discussion}

\subsection{Outputs from Step Signals in the Entry Stream}

To explain the results of these models, dimensionless variables for time, concentration and temperature are defined according to the following definitions (see the list of symbols at the end of the paper): 


$$
\tau=\frac{\text { Elapsed time }}{\text { Mean residence time }}=\frac{t}{\bar{t}}
$$

The mean residence time for the tundish is obtained by dividing the volume of the fluid in the tundish by the volumetric flow rate as follows:

$$
\bar{t}=\frac{V}{Q}
$$

The dimensionless concentration for the step input technique is determined by dividing the actual concentration of the tracer at the outlet by the average concentration of the tracer in the tundish as follows:

$C_{\tau}=$

Concentration of tracer in the exit stream

Calculated mean concentration of tracer in the tundish

The calculated mean concentration is obtained by dividing the injected amount of tracer by the volume of the fluid in the tundish:

$$
C_{\text {mean }}=\frac{\text { Amount of tracer injected }}{\text { Total volume of fluid in tundish }}
$$

The normalized temperature for the step method is simply the fraction of the change from the initial tundish temperature to the subsequent tundish temperature:

$$
F_{\tau}=1-\frac{\left(T_{\mathrm{m}}-T_{\mathrm{f}}\right)}{\left(T_{\mathrm{i}}-T_{\mathrm{f}}\right)}
$$

For the concentration change of a tracer input the Dispersion Model, which is well documented in various textbooks, ${ }^{20,21)}$ was employed. The mathematical expression of this model is given by:

$$
C_{\tau}=\frac{1}{\sqrt{4 \Pi \tau\left(\frac{D_{\mathrm{i}}}{u L}\right)}} \exp \left[\frac{-(1-\tau)^{2}}{4 \tau\left(\frac{D_{\mathrm{j}}}{u L}\right)}\right]
$$

On the other hand, it is possible to demonstrate that the dimensionless fractional temperature change of this model can be expressed by:

$$
F_{\tau}=\frac{1}{2}\left[1-\operatorname{erf}\left(\frac{(1-\tau)}{\sqrt{4 \tau\left(\frac{D_{\mathrm{i}}}{u L}\right)}}\right)\right]
$$

From these equations the distribution of dimensionless concentration is plotted against the dimensionless time resulting that this concentration is a function that only depends on the dispersion parameter $\left(D_{\mathrm{i}} / u L\right)$, which is also the case for the fractional temperature. The value of this parameter is obtained from a variance analysis of the concentration-time experimental curves, additionally, the dispersed plug flow, completely mixed flow and the dead volume of the fluid in the tundish are readily obtained using the standard mathematical techniques. $^{20,21)}$

Figure 3(a) shows the dimensionless concentration-
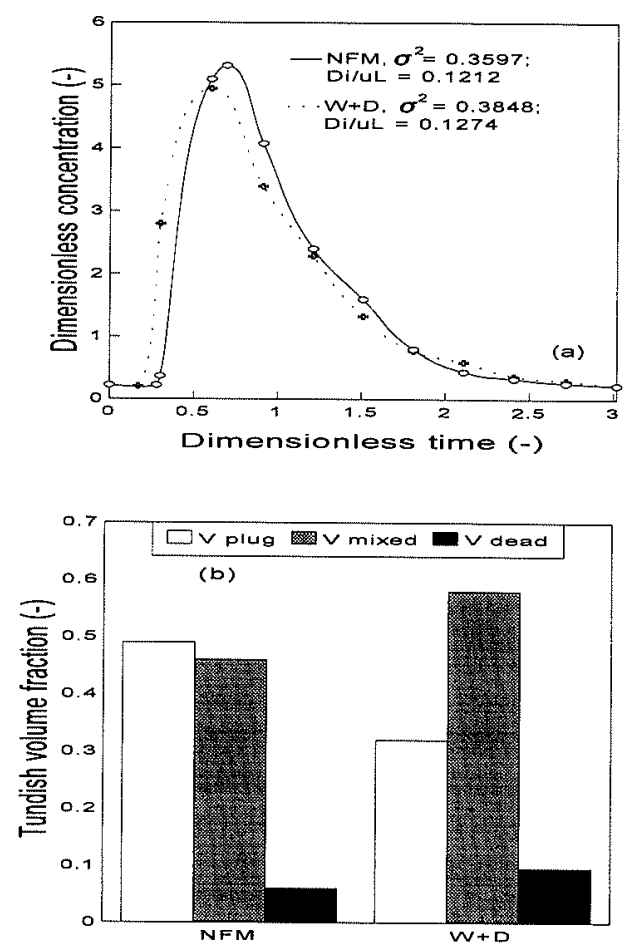

Fig. 3. (a) Concentration readings representing the continuous response to a pulse input of a tracer injected to the fluid entering the tundish. (b) Dispersed plug volume fraction, mixed volume fraction and dead volume fraction for NFM and W+D cases.

time curves for experiments without flow modifiers and with flow modifiers (weir + dam), hereinafter simply called as NFM and $W+D$ respectively. The dispersion parameter for the NFM case was 0.1212 and increased slightly to 0.1274 for the $\mathrm{W}+\mathrm{D}$ case.

Figure 3(b) shows the tundish volume fractions for both experiments. As its may be expected, the completely mixed volume increases for the $W+D$ experiment in relation to the NFM case, these changes are accompanied by a decrease in the plug volume in the first case in relation to the latter. Moreover, the presence of a weir and a dam seems to increase the dead volume presumably because of the formation of stagnant flow zones in the neighborhoods of these devices.

Dimensional temperature-time curves for NFM and $W+D$ experiments are presented in Figs. 4(a) and 4(b) respectively, these experimental data are compared with the results of the mathematical model assuming that the losses of energy through the walls of the model, during the experimental time, are negligible, i.e., adiabatic conditions and, as it is seen, both type of data keep an excellent agreement for both cases. Also, in the same figures the calculations employing the dispersion model are shown and is evident that this model fails to predict the experimental reading temperatures with the accuracy that the mathematical model does. This indicates that an uniaxial dispersion parameter is not enough to describe the flow behavior of the fluid in the tundish.

The corresponding dimensionless temperature-time curves for NFM and $\mathrm{W}+\mathrm{D}$ experiments are shown in Figs. 5(a) and 6(a) respectively. Naturally the mathematical model and the dispersion one keep the same behavior 


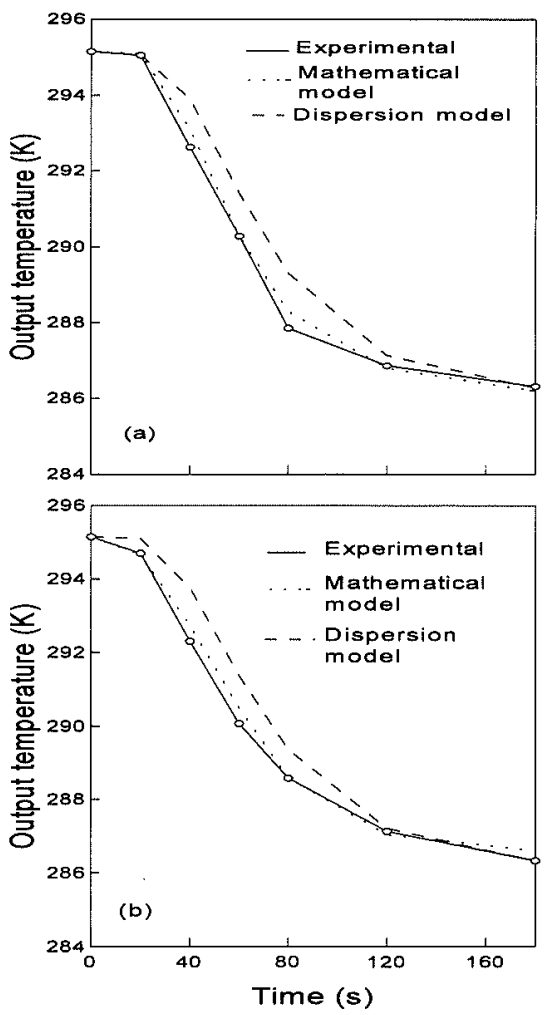

Fig. 4. (a) Experimental temperature of water model compared with calculations made by mathematical and dispersion models for an entry thermal step signal for NFM case. (b) Same as (a) for W+D case
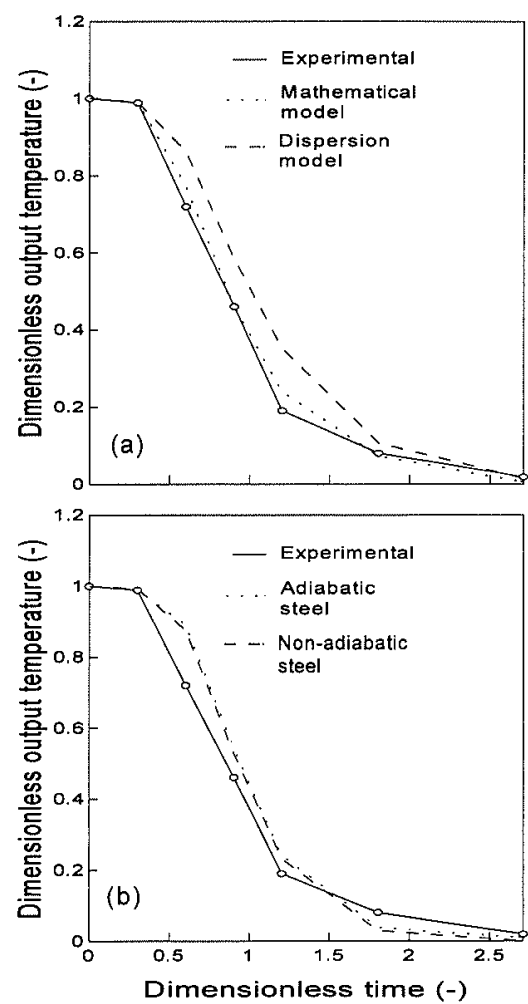

Fig. 5. (a) Dimensionless temperature of water model compared with calculations made by mathematical and dispersion models for an entry thermal step signal for NFM case under adiabatic conditions. (b) Dimensionless temperature for water model compared with steel under adiabatic and non-adiabatic conditions.
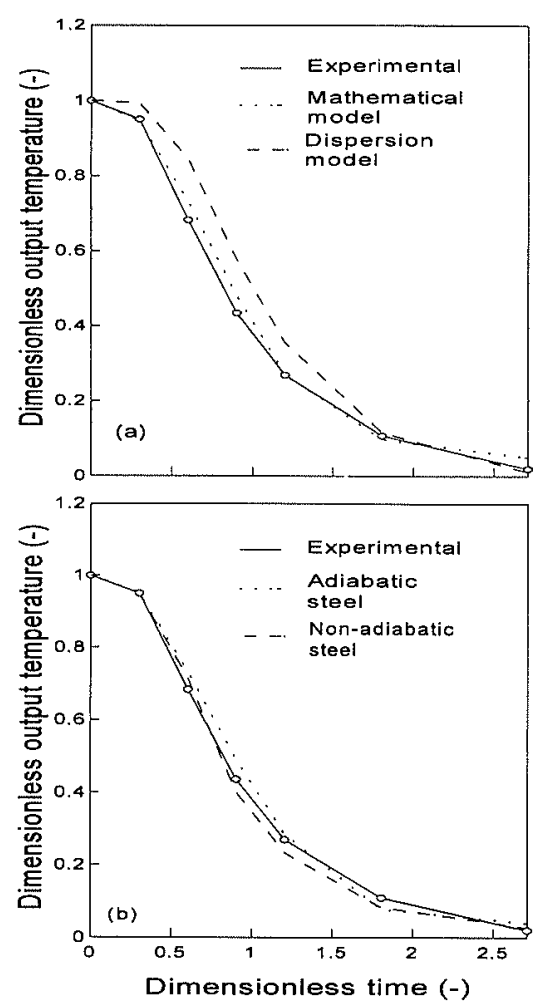

Fig. 6. (a) Dimensionless temperature of water model compared with calculations made by mathematical and dispersion models for an entry thermal step signal for $W+D$ case under adiabatic conditions. (b) Dimensionless temperature for water model compared with steel under adiabatic and non-adiabatic conditions. already described in regard to the experimental data expressed by the dimensional variables. However, one of the most important questions in physical modeling is if this approach is suitable enough to describe the thermal behavior of liquid steel flow in tundishes.

To answer, in part, that question and as far as the mathematical model proved to be very good to predict the overall thermal behavior of water in the tundish model it is assumed that it gives the same level of performance in the prediction of the corresponding thermal behavior of liquid steel in the prototype of the tundish (see the specific conditions in Tables 3 and 4).

In other words, in the absence of experimental temperature data for the prototype, the results provided by the mathematical model will be considered as such. This approach will allow someone to make direct comparisons between the results of the water model and those corresponding to the assumed true thermal behavior of the liquid steel in the prototype in terms of dimensionless as well as dimensional variables.

The results of this procedure are also shown in Figs. 5(b) and 6(b), for NFM and W+D cases, where the calculated curves for liquid steel in the tundish prototype under adiabatic and non-adiabatic conditions are respectively drawn. In this regard, some comments are worthy to be done:
(1) The physical model predicts results which are closer to those of liquid steel under non-adiabatic conditions for NFM and $\mathrm{W}+\mathrm{D}$ schemes. This means that the physical modeling of the thermal bahavior of steel in the prototype would be a valid technique at least in the case of the output response to step inputs of temperature coming in the entry stream.

(2) The results of the water model are slightly closer to the calculations for the W+D scheme. Presumably, this is due to the increase of backmixing flow, besides, the results of the model are slightly below the calculations under adiabatic conditions in almost all the range of time. Differently, the results of the model are slightly lower than the calculations under non-adiabatic conditions in the first part of the time range and slightly higher in the later part.

(3) In all cases the dispersion model observes considerable differences with respect of the results of the experimental results of water and mathematical model.

(4) The presence of flow modifiers slows down the drop rate of the output temperature by increasing the residence time of the fluid in the tundish model.

Then, it can be said that the physical modeling is an acceptably approach to simulate the output response of liquid steel flows in tundishes coming from step input signals in the entry stream. The increase of backmixing 
of the fluid inside the tundish, owing to the presence of flow modifiers, makes this approximation more accurate.

\subsection{The Mathematical Simulation of Steel Flow at Microscopic Levels}

According to the previous section, water model and the mathematical model of steel flow in a prototype agree reasonably well in the global response to step input signals of temperature changes. However, there are doubts about how much the water model is able to simulate the steel flow at microscopic levels, since, it is a common sense that the orientation and magnitude of the velocity vectors play a very important influence on the floatation and the absorption of the non-metallic inclusions by a slag tundish.

Moreover, the direction of steel flow is controlled by flow modifiers and is also influenced by the nonisothermal nature of the tundish due to the buoyancy forces originating from differences of temperature of steel inside the tundish. This stresses the need to investigate the degree of agreement between steel flow in tundishes and non-isothermal water models.

Consequently, here, based in the results previously presented, it is assumed once more that the mathematical model has the capability to predict with good accuracy the true behaviors of fluid flow of water in the model and that of steel in the prototype. Under this con- sideration is then possible to compare the fluid flow of water in the model and steel in the prototype at microscopic levels. In the next lines a series of simulation results will be presented.

\subsubsection{Fluid Flow under Adiabatic Conditions}

For comparison purposes, simulation at a dimensional time in water model of $60 \mathrm{~s}$ after the introduction of the step input temperature (see Fig. 4) will be discussed. This time is equivalent to $139 \mathrm{~s}$ in the prototype. Since the fluid flow is of more interest in the case of flow modifiers than that without these devices, this will be the main case to be considered in the following discussion.

Figures 7(a)-7(c) show the velocity vectors of steel flow inside the prototype under adiabatic conditions, i.e., no heat flow through the tundish walls and the free surface of liquid, at the vertical plane of symmetry and at the vertical planes $z / w=0.6$ and 0.8 respectively. At the plane of symmetry, there is a strong mixing due to the incoming stream of steel, the presence of a dam creates a flow with a well defined orientation toward the outlet nozzle direction.

The weir located in the last third of the tundish length accelerates the steel flow to the right side of the tundish but the existence of the right transverse wall promotes a strong backmixing obtaining a well defined recirculating flow pattern in this part of the tundish.
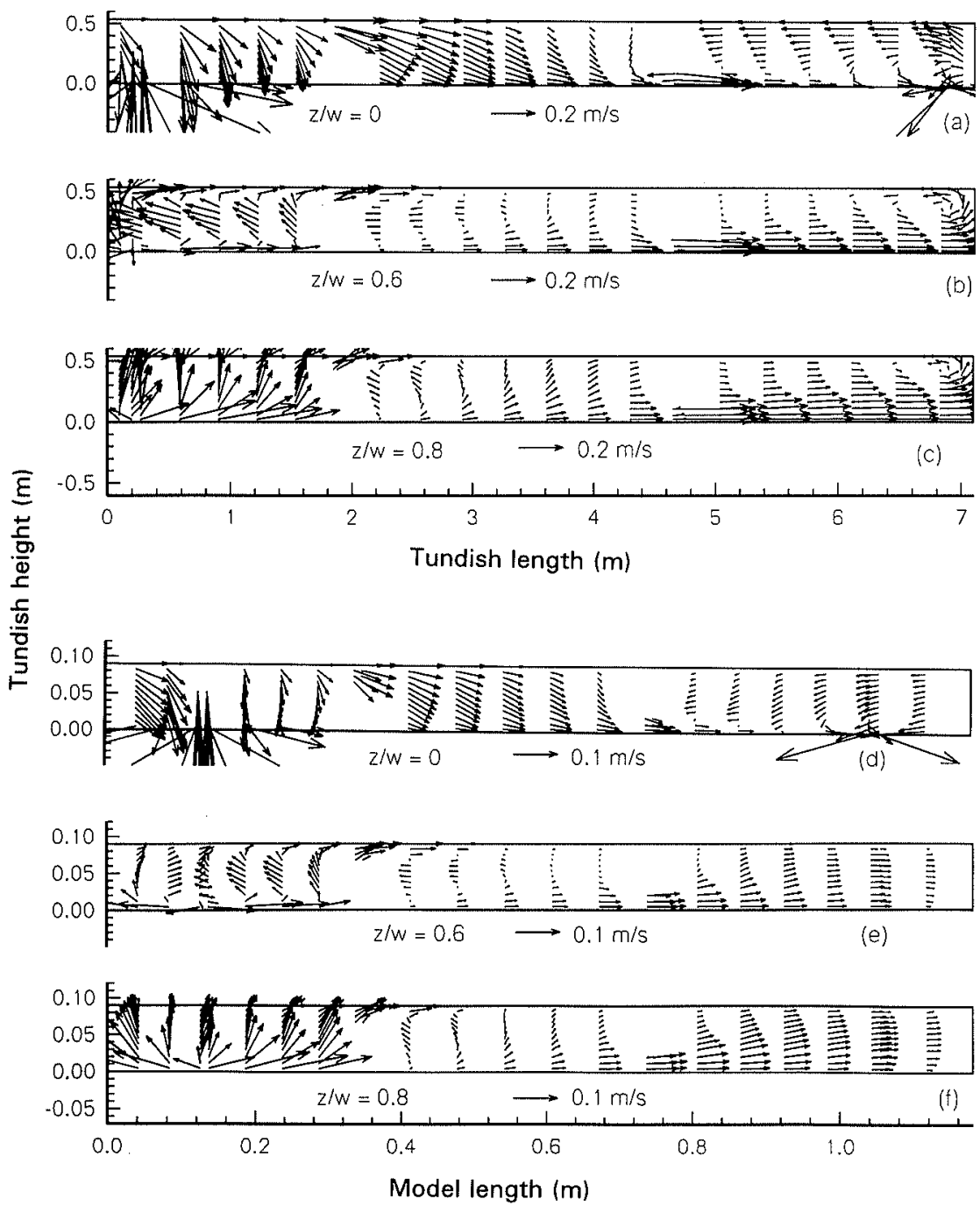

Fig. 7.

(a), (b), (c) Velocity fields of steel in the prototype at different vertical planes after $139 \mathrm{~s}$ of an entry thermal step signal under adiabatic conditions.

(d), (e), (f) Velocity fields of water in the model at the same vertical planes after $60 \mathrm{~s}$ of an entry thermal step signal under adiabatic conditions. 
At the vertical plene $z / w=0.8$ the incoming stream of steel causes a vertical flow outward the surface of the liquid. The fluid flow at the vertical plane $z / w=0.6$, located between the plane of symmetry and the plane at $z / w=0.8$, shows different flow pattern, the outward flow of liquid steel is smaller than the plane nearer to the tundish wall. There is a small recirculating flow located at the right upper side of the tundish similarly to the other two planes already examined.

The corresponding velocity vectors for water in the model of the tundish at an equivalent time of $60 \mathrm{~s}$ are shown in Figs. 7(d), 7(e) and 7(f) for the vertical plane of symmetry and the vertical planes located at $z / w=0.6$ and 0.8 respectively.

Matching these three figures with the corresponding three precedent ones it is clear that the flow of water keeps similarity with the flow of steel in the prototype. The magnitude of the velocity vectors in the case of the water model are, obviously, smaller than those of steel since, as it was pointed out, the Reynolds similarity was not maintained in the present water model. Thus, from the pointview of fluid flow, non-isothermal water models are able to provide an approximate description of microscopic flows of steel in tundishes if these are considered to behave adiabatically. The temperature field of liquid steel in the prototype under adiabatic conditions are shown in Figs. 8(a), 8(b) and 8(c) for the vertical planes at the symmetry position, $z / w=0.6$ and 0.8 respectively expressed by isothermal lines written in Kelvin degrees and as the fractional temperature changes (in parenthesis) from the step input shown in Table 4 and defined by Eq. (7) after $139 \mathrm{~s}$ that the thermal signal was introduced in the tundish. The main characteristics of these termal fields are as follows:

(1) The highest temperatures are located in the stagnant flow zones formed by the dam and the weir.

(2) As it would be expected, at this time, the lowest temperatures are located in the middle of the tundish length this is, evidently, a natural effect due to the colder steel stream coming from the entry point. In the zone located between the dam and the weir the temperature is almost constant for the three vertical planes examined.

(3) The isothermal lines in the three planes examined remain, practically, in the same positions.

On the other hand, Figs. 8(d), 8(e) and 8(f) show the isothermal lines of water in the model of the tundish expressed in the same units, Kelvin degrees and fractional temperature changes. This later way of expression permits to make a direct comparison of Figs. 8(a), 8(b) and $8(\mathrm{c})$ with Figs. $8(\mathrm{~d}), 8(\mathrm{e})$ and $8(\mathrm{f})$, the comments on this are as follows:

(1) Differently to the results of steel flow in the prototype, the positions of the isothermal lines as well as their shapes, in the plane of symmetry and those in the planes at $z / w=0.6$ and 0.8 do not coincide. Even in the cases of these two later vertical planes the isother-
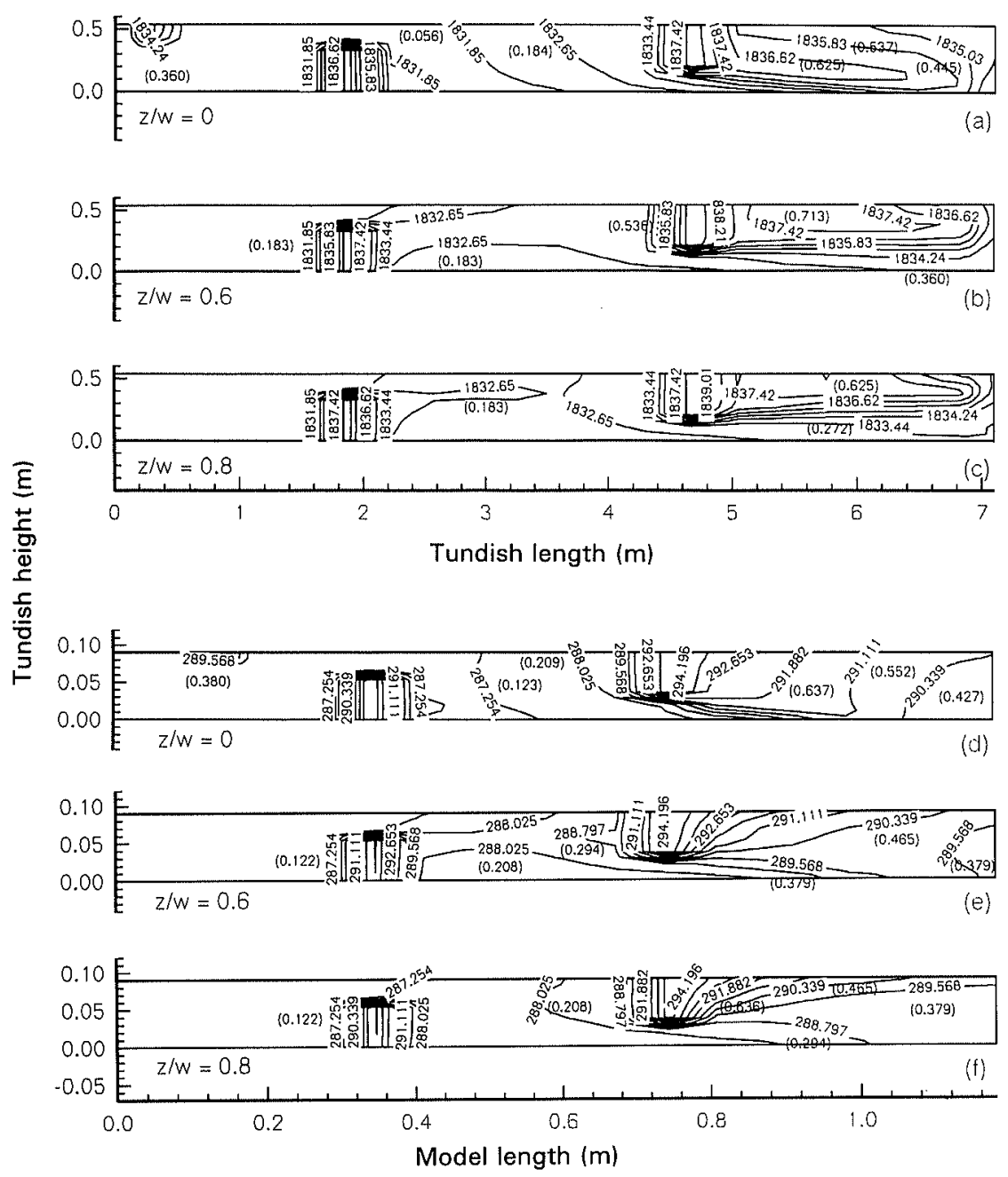

Fig. 8.

(a), (b), (c) Isothermal and isodimensionless temperature lines of steel in the prototype under adiabatic conditions at different vertical planes after $139 \mathrm{~s}$ of an entry thermal step signal.

(d), (e), (f) Same as (a), (b), (c) for water in the model. 
mal lines, although maintain a similar shape, their positions are different.

(2) The magnitude of the temperatures follow, generally, the same pattern of that already described for the prototype, the highest temperatures are also located in the stagnant points of the model originated by the presence of the dam and the weir.

(3) The overall positions of the fractional thermal lines for water in the model are similar to those corresponding to the steel in the prototype.

From this analysis is clear that, in spite of the quite different physical properties of water and steel, the behavior of the flow of steel in the prototype is well simulated by the water model.

\subsubsection{Fluid Flow under Non-adiabatic Conditions}

When the heat flows through the walls and the free surface are taken into account in the boundary conditions of this mathematical model, a condition described here as non-adiabatic, the flow of steel as well as its thermal behavior suffer considerable changes. Figures 9(a), 9(b) and 9(c) show the velocity vectors and Figs. 9(d), 9(e) and $9(f)$ the isothermal lines of liquid steel inside the prototype of the tundish for the three referred vertical planes calculated at a time of $139 \mathrm{~s}$.

Comparing Figs. 9(a), 9(b) and 9(c) for steel flow with Figs. 7(d), 7(e) and 7(f) for water respectively, it is easily observed that there are considerable differences between both sets of simulation results for the already defined three vertical planes.

At the plane of symmetry for steel and water Figs. 9(a) and 7(d) respectively, the flow pattern of both fluids maintain similar structures from the entry point up to the position of the dam.

Differences arise in the last third of the tundish length, in the case of steel, the high velocities are constrained to the proximities of the bottom of the tundish and, approximately, in the last two thirds of the liquid height the liquid steel flow remains practically stagnant with the exception of a small zone of recirculating flow formed by the presence of the transversal right wall of the tundish. In contrast, as was already described, the flow of water presents a strong flow, clearly directed toward the outlet nozzle, through all the last third of the tundish length.

For the planes $z / w=0.6$ and 0.8 there is also a similar pattern of fluid flow for both fluids in the first two thirds of the tundish length up to the position of the dam. In the case of steel (Figs. 9(b) and 9(c)), a strong recirculating flow is formed with the highest velocities located close the bottom of the tundish. This is not the characteristic flow of water in the model (Figs. 7(e) and 7(f)) where there is not an appreciable recirculation of this fluid.

It is evident that the observations mentioned above are also applicable if the results of the mathematical simulation results of steel in the prototype under adiabatic conditions (Figs. $7(\mathrm{~d}), 7(\mathrm{e})$ and $7(\mathrm{f})$ ) are compared with the simulation results of steel in the prototype under non-adiabatic conditions (Figs. 9(a),
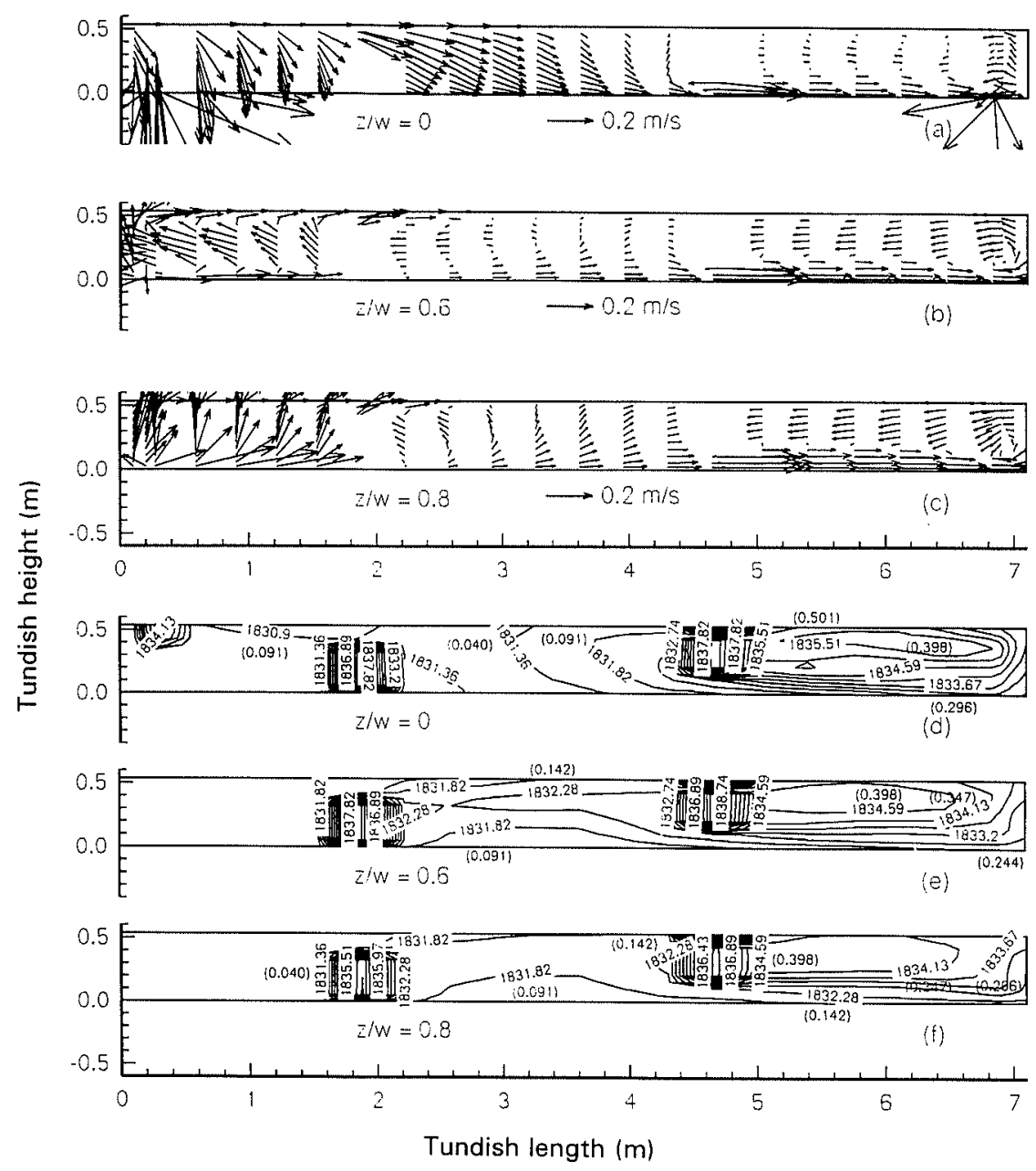

Fig. 9.

(a), (b), (c) Velocity fields of steel at different vertical planes after $139 \mathrm{~s}$ of an entry thermal step signal in the tundish calculated under non-adiabatic conditions. (d), (e), (f) Isothermal lines and isodimensionless temperature lines for the cases shown in (a), (b) and (c) respectively. 
9(b) and 9(c)).

From those results, it is inferred that the temperature fields should be completely different between the water model and the steel prototype. Indeed, Figs. 9(d), 9(e) and $9(\mathrm{f})$ show the temperature field, expressed by isothermal lines, of steel in the prototype which should be compared with Figs. 8(d), 8(e) and 8(f) of water in the model repectively. At the plane of symmetry (Figs. 9(d) and 8(d)), the thermal fields in terms of the fractional temperature changes are no equivalent. Again, the temperature of steel between the dam and the weir is, rather, uniform and is already very close to the final temperature of $1831 \mathrm{~K}$. In the case of water, although its temperature is near to be uniform, is still far from the final temperature of $286.73 \mathrm{~K}$ and this is due to the adiabatic nature of the model under the established assumptions in this paper.

It is also worthy to be noticed that the shapes of the isothermal lines are also no equivalent between the model and the prototype. Similar comments can be made in relation to the thermal field of the steel flow at the vertical planes $z / w=0.6$ and 0.8 .

Comparing now Figs. 9(d), 9(e) and 9(f) among themselves it is found that the liquid steel has a rather uniform temperature through all the tundish volume.

From these descriptions is clear that modeling the non-isothermal flow of steel in a prototype by adiabatic-non-isothermal water models may lead to uncorrect conclusions about the orientation of velocity vectors and the general structure of the flow. The non-isothermal water model does not predict the magnitude neither the position of the velocity and temperature fields in the prototype under non-adiabatic conditions.

The reason for the existence of very similar flow patterns of steel under adiabatic and non-adiabatic conditions (see and compare Figs. 9(a), 9(b) and 9(c) with Figs. 7(a), 7(b) and 7(c) respectively) between the entry point and the dam is because of the very high inertial momentum transfer promoted by the incomingstream of steel. This means that the momentum originated by the differences of temperature, which by the way, are smaller compared to those in the last third of the tundish length, give place to buoyancy forces that are negligible compared to the inertial ones.

The influence that the results of these predictions may bring about on the movement and the trajectories of non-metallic inclusions is not clarified at this stage. However, what can be said is that non-isothermal water models should be employed with caution if steel cleanliness studies in the tundish is the aim of a research. To elucidate this, the authors continue this research to model physically and mathematically the trajectory of non-metallic inclusions under non-isothermal and non-adiabatic conditions to understand the effects of fluid flow of steel in the tundish on its cleanliness which will be the subject of future communications.

As a reference, the fluid structure and the thermal field of steel in the tundish, without flow modifiers, at a time of $139 \mathrm{~s}$ after the start of the step input signal of temperature are presented in Figs. 10(a) and 10(b) at the plane of symmetry respectively. There are not zones with recirculating flows of steel, the flow follows a monotonous trajectory toward the oulet nozzle direction. The contrasty between the flows with and without flow modifiers is more clearly seen by comparing Fig. 10(a) with Fig. 7(a). On the other hand the temperature field of Fig. 10(b) shows that the temperature of steel inside the tundish decreases to values that are lower than that of the coming stream $(1831 \mathrm{~K})$. Such a behavior is due to the non-adiabatic effects and this is clearly seen by observing Figs. 10(b) and 8(b).

\section{Conclusions}

Steel flow and heat transfer in the tundish have been physically and mathematically modeled and the following are the main conclusions:

(1) The mathematical model predicts with an excellent agreement the outlet temperature response to step input temperatures in the inlet stream of water in the tundish model.

(2) The dispersion model is not able to predict satisfactorily the outlet temperature response to step temperatures in the inlet stream of water in the tundish model.

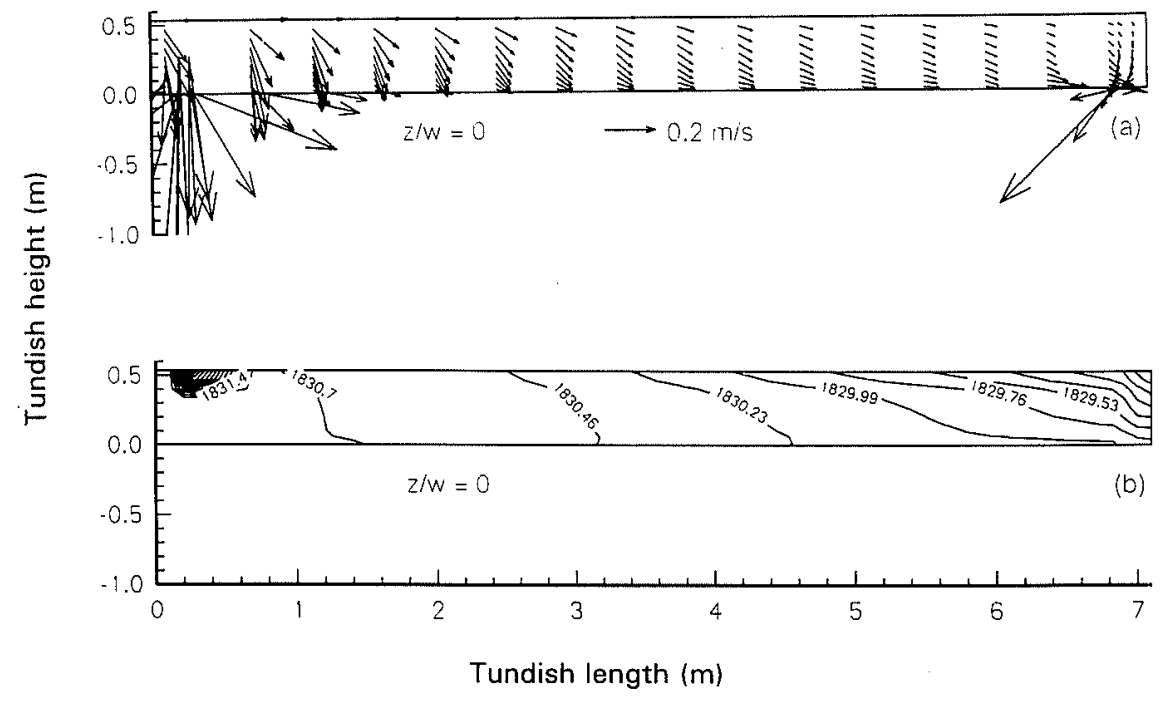

Fig. 10.

(a) Velocity field of steel flow in the prototype after $139 \mathrm{~s}$ of an entry thermal step signal in the tundish calculated under non-adiabatic conditions without flow modifiers

(b) Isothermal lines for the case shown in (a). 
(3) In direct matching comparisons of mathematical simulations for the water model and steel in the prototype the specific conclusions are;

a) The fluid flow pattern of water in the model calculated under adiabatic conditions is similar to that of steel flow calculated under the same conditions. The thermal fields between both systems are different but follow the same tendency, although, their shapes are different.

b) The fluid flow pattern of water in the model calculated under adiabatic conditions is quite different to that of steel flow calculated under, more realistic, nonadiabatic conditions. As a consequence, the thermal fields in both systems are also different.

(4) On basis of the precedent conclusions it is recommended to have caution in using water models to simulate the dynamic behavior of steel flow and the dynamic behavior of non-metallic inclusions in tundishes of steel.

\section{Acknowledgements}

The authors give the thanks to Consejo Nacional de Ciencia y Tecnología (CoNaCyT) for their financial support to this research. Also, the authors feel very indebted to the institutions SNI, COFAA, Instituto Politécnico Nacional and Instituto Tecnológico de Morelia for their permanent assistance to research activities in the Process Metallurgy field.

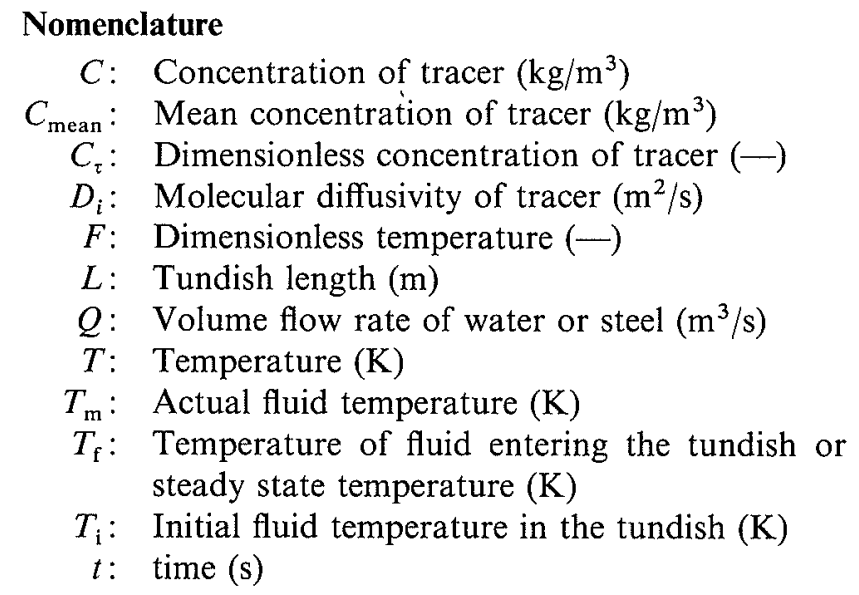

$\bar{t}:$ mean residence time (s)

$u$ : Water velocity in the axial direction of the tundish model $(\mathrm{m} / \mathrm{s})$

$V:$ Fluid volume in the reactor $\left(\mathrm{m}^{3}\right)$

$\varepsilon$ : Dissipation rate of turbulence kinetic energy $\left(\mathrm{m}^{2} / \mathrm{s}^{3}\right)$

$\tau$ : Dimensionless time $(-)$

\section{REFERENCES}

1) F. Kemeny, D. J. Harris, A. Mclean, T. R. Meadowcroft and J. D. Young: Proc. 2nd. PTD Conf., Vol. 2, ISS, Pittsburgh, (1981), 232.

2) R. Ahuja and Y. Sahai: Proc. 24th Annual Conf. of Metallurgists, CIM, Canada, (1985), 73.

3) Y.Sahai and R. Ahuja: Ironmaking Steelmaking, 13(1986), 241.

4) M. Hashio, M. Toduka, M. Kawasaki and T. Watanabe: Proc. 24th Conf. of Metallurgists, CIM, Canada, (1985), 65.

5) C. J. Dobson, R. Serje and K. Gregory: Proc. 4th Int. Conf. on Continuous Casting, Inst. Met., England, (1988), 682.

6) K. Y. M. Lal, M. Saculdean, S. Tanaka and R. I. L. Guthrie: Metall. Trans., 17B (1986), 449.

7) J. Szekely, O. J. Olegbusi and N. El-Kaddah: $P C H$ Physico Chemical Hydrodynamics, 9 (1987), 453.

8) H. E. Youduo and Y. Sahai: Acta Metall. Sin., 1, 2 (1988), 93.

9) J. de J. Barreto S., A. W. D. Hills and D. M. Allen Booth: Proc. PTD Conf. on Continuous Casting 13, ISS, Nashville, (1995), 253.

10) M. L. Lowry and Y. Sahai: Proc. Steelmaking Conf., 74, ISS, Washington D.C., (1991), 505.

11) S. Chakraborty and Y. Sahai: ISIJ Int., 31 (1991), 960.

12) S. Chakraborty and Y. Sahai: Ironmaking Steelmaking, 19 (1992), 479.

13) S. Chakraborty and Y. Sahai: Ironmaking Steelmaking, 19 (1992), 488.

14) L. J. Heaslip, A. Maclean and I. D. Sommerville: Fluid Flow in Continuous Casting, Vol. 1, Chapter 2, ISS, USA, (1983), 67.

15) J. Szekely: Fluid Flow Phenomena in Metals Processing, Academic Press, N.Y., (1979).

16) J. L. Yeh, W. S. Hwang and C. L. Chou: ISIJ Int., 33 (1993), 588.

17) B. E. Launder and D. B. Spalding: Mathematical Models of Turbulence, Academic Press, N.Y., (1972).

18) R. I. Issa: J. Computational Physics, 62 (1985), 405.

19) R. I. Issa, B. Ahmadi-Befrui, K. R. Beshay and A. D. Gossman: J. Computational Physics, 93 (1991), 388.

20) O. Levenspiel: Chemical Reaction Engineering, 2nd Ed., Wiley Int., New York, (1972).

21) D. M. Himmelblau: Process Analysis and Simulation, John Wiley and Sons, New York, (1968). 